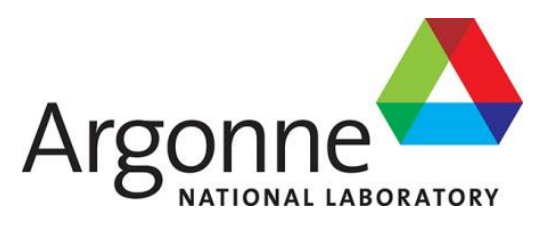

\title{
Evaluation of the OpenCL AES Kernel Using the Intel FPGA SDK for OpenCL
}

Argonne Leadership Computing Facility 


\section{About Argonne National Laboratory}

Argonne is a U.S. Department of Energy laboratory managed by UChicago Argonne, LLC under contract DE-AC02-06CH11357. The Laboratory's main facility is outside Chicago, at 9700 South Cass Avenue, Argonne, Illinois 60439. For information about Argonne

and its pioneering science and technology programs, see www.anl.gov.

\section{DOCUMENT AVAILABILITY}

Online Access: U.S. Department of Energy (DOE) reports produced after 1991 and a growing number of pre-1991 documents are available free via DOE's SciTech Connect (http://www.osti.gov/scitech/)

\section{Reports not in digital format may be purchased by the public from the} National Technical Information Service (NTIS):

U.S. Department of Commerce

National Technical Information Service

5301 Shawnee Rd

Alexandria, VA 22312

www.ntis.gov

Phone: (800) 553-NTIS (6847) or (703) 605-6000

Fax: (703) 605-6900

Email:orders@ntis.gov

Reports not in digital format are available to DOE and DOE contractors from the Office of Scientific and Technical Information (OSTI):

U.S. Department of Energy

Office of Scientific and Technical Information

P.O. Box 62

Oak Ridge, TN 37831-0062

www.osti.gov

Phone: (865) 576-8401

Fax: (865) 576-5728

Email: reports@osti.gov

\section{Disclaimer}

This report was prepared as an account of work sponsored by an agency of the United States Government. Neither the United States Government nor any agency thereof, nor UChicago Argonne, LLC, nor any of their employees or officers, makes any warranty, express or implied, or assumes any legal liability or responsibility for the accuracy, completeness, or usefulness of any information, apparatus, product, or process disclosed, or represents that its use would not infringe privately owned rights. Reference herein to any specific commercial product, process, or service by trade name, trademark, manufacturer, or otherwise, does not necessarily constitute or imply its endorsement, recommendation, or favoring by the United States Government or any agency thereof. The views and opinions of document authors expressed herein do not necessarily state or reflect those of the United States Government or any agency thereof, Argonne National Laboratory, or UChicago Argonne, LLC. 


\section{Evaluation of the OpenCL AES Kernel Using the Intel FPGA SDK for OpenCL}

prepared by

Zheming Jin, Kazutomo Yoshii, Hal Finkel, Franck Cappello

Argonne Leadership Computing Facility, Argonne National Laboratory

April 20, 2017 


\section{Evaluation of the OpenCL AES Kernel using the Intel FPGA SDK for OpenCL}

\section{Introduction}

The OpenCL standard is an open programming model for accelerating algorithms on heterogeneous computing system. OpenCL extends the C-based programming language for developing portable codes on different platforms such as CPU, Graphics processing units (GPUs), Digital Signal Processors (DSPs) and Field Programmable Gate Arrays (FPGAs). The Intel FPGA SDK for OpenCL is a suite of tools that allows developers to abstract away the complex FPGA-based development flow for a high-level software development flow. Users can focus on the design of hardware-accelerated kernel functions in OpenCL and then direct the tools to generate the low-level FPGA implementations. The approach makes the FPGA-based development more accessible to software users as the needs for hybrid computing using CPUs and FPGAs are increasing. It can also significantly reduce the hardware development time as users can evaluate different ideas with high-level language without deep FPGA domain knowledge.

The Advanced Encryption Standard (AES) specifies a standard encryption algorithm that is used worldwide to protect electronic data. The OpenCL AES kernel was originally developed by Liu et al. [1] at Virginia Tech. The kernel function converts data to an unintelligible form using cryptographic keys. The kernel is constructed as a dynamic library engine that can be linked into the OpenSSL framework. The authors evaluated the performance of the kernel on the scalable multi-FPGA architecture [2]. Their hardware platform is based on the M506 module with a StratixV A3 FPGA and 8GB DDR3 memory. On a single M506 module, they achieved the highest FPGA throughput of 5.1Gbps (Giga bits per second) using the OpenCL SIMD4 vectorization optimization. The throughput of the same test is $4.4 \mathrm{Gbps}$ on an $17-4770 \mathrm{~K}$ processor.

In this report, we evaluate the performance of the kernel using the Intel FPGA SDK for OpenCL and Nallatech 385A FPGA board. Compared to the M506 module, the board provides more hardware resources for a larger design exploration space. The kernel performance is measured with the compute kernel throughput, an upper bound to the FPGA throughput. The report presents the experimental results in details. The Appendix lists the kernel source code.

\section{Overview of the two FPGA devices}

FPGA offers a wide variety of configurable memories, high-speed I/Os, logic blocks and routing. StratixV and Arria10 series of Intel FPGAs are two products for highperformance applications. Table 1 compares the technology and resource counts of the Stratix V A3 FPGA device [3] on the M506 with those of the Arria10 GX1150 FPGA device [4] on the Nallatech 385A. The Arria10 device features 20-nm SoC process technology and operates at $0.95 \mathrm{~V}$ core voltage while the StratixV device uses 28-nm technology and operates at $0.9 \mathrm{~V}$. Based on the results in Table 1, the Arria10 device has 
approximately $3 \mathrm{X}$ more resources than the StratixV device, so it provides a larger design exploration space for the performance evaluation of the AES kernels.

Table 1. Device overview of two FPGAs

\begin{tabular}{|l|c|c|c|c|c|}
\hline \multicolumn{1}{|c|}{ Device } & Technology & Logic elements & ALMs & Register & $\begin{array}{c}\text { M20K } \\
\text { memory bits }\end{array}$ \\
\hline $\begin{array}{l}\text { Stratix } \\
\text { 5SGXMA3 }\end{array}$ & $28 \mathrm{~nm}$ & $340 \mathrm{~K}$ & 128,300 & $513 \mathrm{~K}$ & $19 \mathrm{Mb}$ \\
\hline $\begin{array}{l}\text { Arria } \\
10 \mathrm{AX} 115\end{array}$ & $20 \mathrm{~nm}$ & $1150 \mathrm{~K}$ & 427,200 & 1708800 & $54260 \mathrm{~Kb}$ \\
\hline
\end{tabular}

\section{Nallatech 385A}

Nallatech 385A is a PCIe-based FPGA accelerator card that features an Arria 10 GX1150 FPGA device, PCIe $\times 8$ Generation 3 host interface, and two banks of 4GB DDR3 memory. The theoretical peak floating-point performance is 1.5 TFLOPS and the theoretical peak memory bandwidth approximately $34 \mathrm{~GB} / \mathrm{s}$.

\section{Kernel optimizations}

As described in [5], users can take advantage of compute unit replication and kernel SIMD vectorization to achieve higher throughput or lower kernel time. The compute device replication generates multiple compute units for each kernel. Each compute unit has its own memory access interface. The SIMD vectorization duplicates only the data path of the compute unit without generating additional memory interfaces. When the kernel is vectorized, the static memory coalescing is performed automatically by the compiler to generate a memory interface that can coalesce the multiple memory loads into a single wide load. While there is no limit to the number of kernel copies that users can specify, the number of SIMD lanes must be a power of two. The compiler will give a warning when the width of all the lanes exceeds the memory interface data width.

\section{Experimental setup}

In this work, a host system is set up with two $2.6 \mathrm{GHz}$ Intel Xeon processors and 32GB DDR3 memory for each node. The PCI Express provides a Gen $2 \times 8$ connection. CentOS 6.8 with Linux kernel 2.6.32 is installed as the operating system. We used the Intel's FPGA SDK for OpenCL version 16.0.2 Pro Prime for the experimental results.

For the kernel test, we choose the same input data size as in the paper [2]. The size of the input data is $2 \mathrm{~GB}$ and the block size is $128 \mathrm{MB}$. The host program divides the input data into 16 blocks $(2 \mathrm{~GB} / 128 \mathrm{MB})$ and they are encrypted sequentially in 16 passes. The test mode is the AES 256-bit algorithm in ECB mode.

\section{Experimental results}

Table 2 lists the FPGA resource usage and the maximum frequency (Fmax) of each kernel. The default kernel is the baseline kernel without any kernel optimization. 
Replication of compute unit is represented as "cuX" where X indicates the replication times. The combination of kernel duplication and N-lane vectorization is represented as "simdN+cuX". The logic utilization of each kernel is below $40 \%$. The RAM block usage increases from $17 \%$ (simd2) to $45 \%$ (simd16) for the kernel vectorization and from $20 \%$ (cu2) to $67 \%$ (cu16) for the kernel duplication. The significant increase in RAM blocks for both optimizations is due to the duplication of the four 256-entry by 32-bit look-up tables in the OpenCL AES kernel. The SDK fails to build when there are more than two duplicate kernels with SIMD16 vectorization, because they require more RAM blocks than the device can provide. As shown in the table, kernel duplication degrades the Fmax from $247 \mathrm{MHz}$ to $186 \mathrm{MHz}$ while kernel vectorization decreases the maximum frequency from $250 \mathrm{MHz}$ to $218 \mathrm{MHz}$. This indicates the impact of increasing RAM blocks on the timing of the kernel implementation.

Table 2. FPGA resource usage and Fmax of the implemented kernels

\begin{tabular}{|l|l|l|l|l|}
\hline Kernel & $\begin{array}{l}\text { Logic } \\
\text { Utilization }\end{array}$ & $\begin{array}{l}\text { Memory } \\
\text { bits }\end{array}$ & $\begin{array}{l}\text { RAM } \\
\text { blocks }\end{array}$ & $\begin{array}{l}\text { Fmax } \\
\text { (MHz) }\end{array}$ \\
\hline default & $13 \%$ & $8 \%$ & $16 \%$ & 245 \\
\hline simd2 & $13 \%$ & $8 \%$ & $17 \%$ & 249 \\
\hline simd4 & $14 \%$ & $9 \%$ & $21 \%$ & 250 \\
\hline simd8 & $15 \%$ & $10 \%$ & $29 \%$ & 238 \\
\hline simd16 & $18 \%$ & $14 \%$ & $45 \%$ & 218 \\
\hline cu2 & $14 \%$ & $8 \%$ & $20 \%$ & 247 \\
\hline cu4 & $17 \%$ & $10 \%$ & $26 \%$ & 233 \\
\hline cu8 & $24 \%$ & $12 \%$ & $40 \%$ & 200 \\
\hline cu16 & $36 \%$ & $17 \%$ & $67 \%$ & 186 \\
\hline simd16+cu2 & $25 \%$ & $21 \%$ & $78 \%$ & 207 \\
\hline
\end{tabular}

The FPGA power consumption fluctuates between $30 \mathrm{~W}$ and $60 \mathrm{~W}$ based on the power meters reading at $100 \mathrm{~ms}$ interval over the time of testing all the kernels. We use the compute kernel throughput (Mbps) to measure the performance of each implementation. The compute kernel throughput is calculated by dividing the bit size of the workload over the total kernel execution time on the FPGA. The kernel execution time on an FPGA is consistent. We found the FPGA throughput, which is calculated by dividing the workload

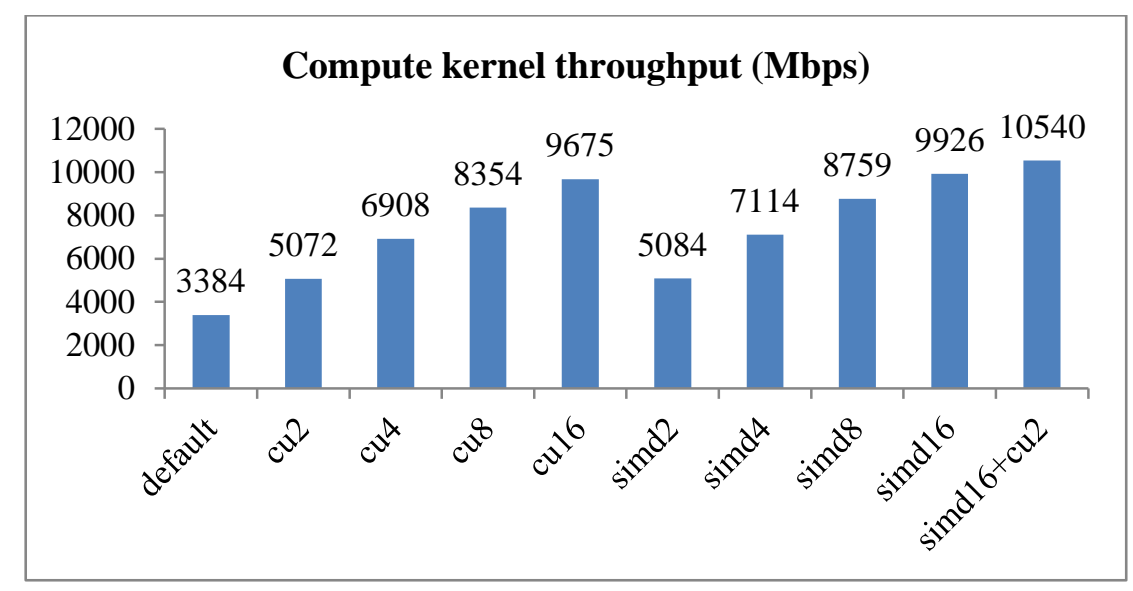

Figure 1 Compute kernel throughput on the Nallatach 385A FPGA board 
over the total execution time, is not consistent for each application run. The total execution time includes the data transfer time between the host and the device, kernel execution time and the host execution time. While the compute kernel throughput is the upper bound to the FPGA throughput, it accurately reflects the performance of each kernel running on an FPGA device.

As shown in Figure 1, the kernel throughput does not increase linearly with the increase of the number of compute units and/or vector lanes. The throughput increases by less than $50 \%$ when the number of compute units or vector lanes doubles from two to sixteen. The throughput of each SIMD implementations is slightly better than that of compute unit duplications.

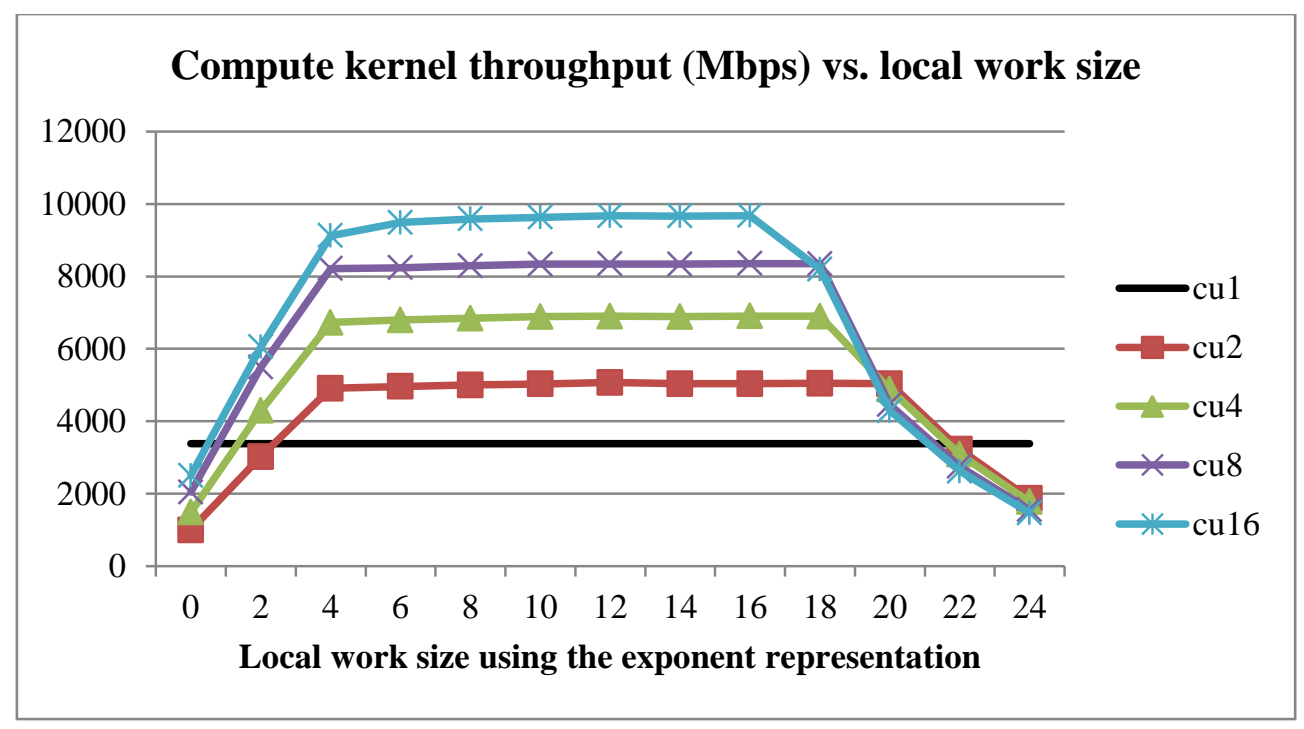

Figure 2 Compute kernel throughput vs. Local work size in kernel duplication

In the kernel duplication optimization, it is interesting that the kernel performance is intimately related with the local work size [6] for multiple compute units. Figure 2 presents the impact of the local work size on the compute kernel throughput. The $x$ axis represents the local work size using the exponent as shorthand for $2^{\text {exponent }}$. The local work sizes are multiples of four. When there is a single compute unit (as a reference), the kernel throughput slightly fluctuates between 3,382 Mbps and 3,384 Mbps over local work sizes. For multiple compute units, the kernel throughput reaches its maximum when the local work size ranges from $2^{10}$ to $2^{16}$.

\section{Conclusion}

This report provides detailed results of the OpenCL AES kernel implemented on a single Arria10 GX1150 FPGA board that is available in the laboratory. In our experiment, the compute kernel throughput is the upper bound to the FPGA throughput for the AES kernel. The results of the compute kernel throughput show the kernel duplication and vectorization improve the kernel performance at the cost of high hardware resources. Using more compute units or vector lanes increases the block RAM usage, which in turn 
degrades the maximum frequency of the FPGA implementations. The best performance using kernel duplication is achieved by experimenting with the local work sizes.

\section{Acknowledgments}

This material is based upon work supported by the U.S. Department of Energy Office of Science, under contract DEAC02-06CH11357.

\section{Reference}

[1] Z. Liu and A. R. M. Ganesh "OpenCL-AES”, Dec. 2011 URL: http://www.github.com/softboysxp/OpenCL-AES.

[2] S. Gao and J. Chritz, "Characterization of OpenCL on a scalable FPGA architecture," 2014

International Conference on ReConFigurable Computing and FPGAs (ReConFig14), Cancun, 2014, pp. 1-

6. doi: 10.1109/ReConFig.2014.7032505

[3] Stratix V Device Overview

[4] Arria 10 Device Overview

[5] Intel FPGA SDK for OpenCL. Programming Guide. UG-OCL002. 2016.10.31

[6] https://www.khronos.org/registry/OpenCL/sdk/1.0/docs/man/xhtml/clEnqueueNDRangeKernel.html

\section{Appendix}

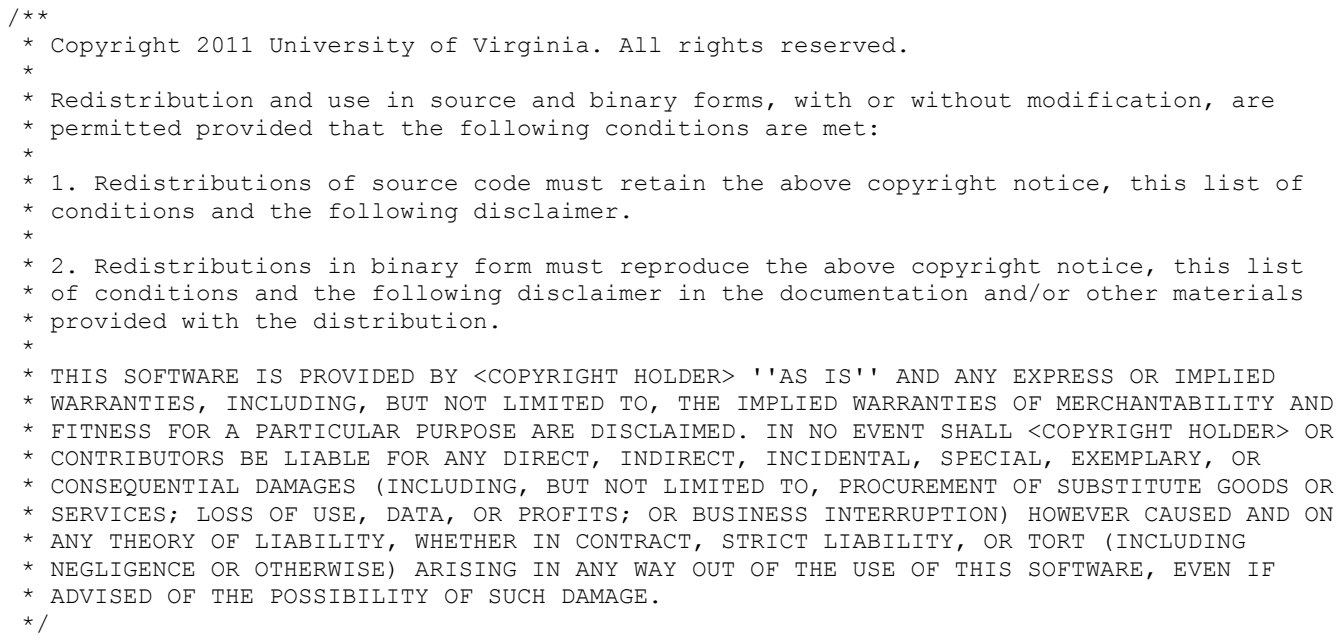

// The array values omitted to save space constant uint $\mathrm{Te} 0[256]=\{\ldots \ldots\}$

constant uint $\operatorname{Te} 1[256]=\{\ldots \ldots\}$

_constant uint $\mathrm{Te} 2[256]=\{\ldots \ldots\}$

constant uint $\operatorname{Te} 3[256]=\{\ldots \ldots\}$

__ kernel void AES_encrypt (__global uint4 *state, __constant uint4 *rk, uint rounds) \{

uint global_id = get_global_id(0);

uint4 s, t; 


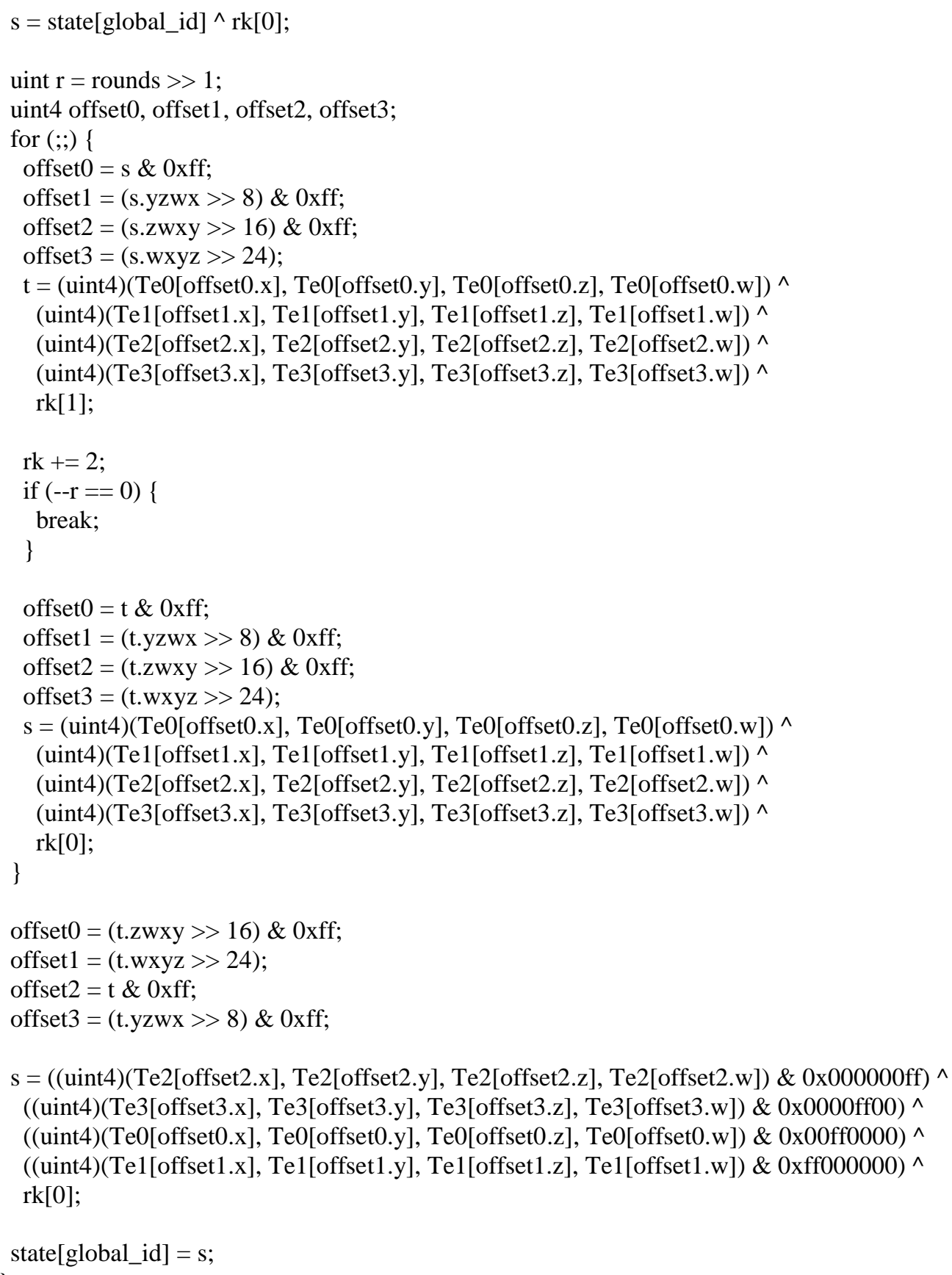




\section{Argonne}

\section{Argonne Leadership Computing Facility}

Argonne National Laboratory

9700 South Cass Avenue, Bldg. 240

Argonne, IL 60439

www.anl.gov 